\title{
International databases open the door to improved care for rare bleeding disorders
}

Flora Peyvandi

The development of registries through international collaboration has facilitated better understanding of the rare bleeding disorders. Such work has shown that rare bleeding disorders are heterogeneous and need to be studied singularly, and that heterozygous patients may bleed. There is a need to understand the minimum plasma coagulant activity level to prevent spontaneous bleeding. Moreover, due to the low prevalence of rare bleeding disorders, the management of this patient population remains a challenge. Data collection on clinical history, efficacyandsideeffectsoftreatmentneedstobeharmonised.

Keywords: rare bleeding disorders, haemophilia, Katharine Dormandy, databases

Rare diseases have a low prevalence and affect a small number of people. In Europe, rare disorders are defined as those affecting no more than 5 people per 10,000 inhabitants; in the United States, the definition set out in the Rare Diseases Act of 2002 is of a disorder affecting less than 200,000 people in the general population [1]. Therefore, in western countries a disease is defined as rare when it has a prevalence of 1 in 1500/2000.

For rare bleeding disorders, the data available in the literature is limited. The World Federation of Hemophilia started collecting information on rare bleeding disorders in 2004. Its survey for 2014 suggested that 275,762 people around the world were affected by a rare bleeding disorder [2]. However, about $40 \%$ of the data were registered in Europe, which suggests a significant under registration for the rest of the world. The available data suggest that about 95-97\% of the patients with a coagulation disorder are affected by haemophilia and von Willebrand disease, and only $3-5 \%$ have a rare disorder [3]. Among the rare bleeding disorders, deficiencies of factor VII and factor XI seem to account for about one third of the patients affected.

The past 20 years have seen increasing investment in rare bleeding disorders, allowing identification of patients by genotype and phenotype diagnosis, as well as improvement of knowledge through specialist networks. In addition, given the substantial improvements in basic and clinical research, more drug companies are beginning to invest in rare bleeding disorders, resulting in more clinical trials and offering the potential to develop more drugs in order to be able to treat these patients better.

Flora Peyvandi, Associate Professor of Internal Medicine at the University of Milan, and Director of the Angelo Bianchi Bonomi Haemophilia and Thrombosis Centre, Fondazione IRCCS Ca' Granda, Ospedale Maggiore Policlinico, Milan, Italy

Email: flora.peyvandi@unimi.it
Table 1: Assigned categories of clinical bleeding severity [4]

Bleeding severity

Asymptomatic

Grade I bleeding

Grade II bleeding

\section{Definition}

No documented bleeding episodes

Bleeding after trauma or drug ingestion

Spontaneous minor bleeding: bruising, ecchymosis, minor wounds, oral cavity bleeding, epistaxis and menorrhagia

Grade III bleeding

Spontaneous major bleeding: haematomas, haemarthrosis, CNS, GI,

and umbilical cord bleeding

My interest in rare bleeding disorders began in 1995 while visiting Tehran the Imam Khomeini Hospital we identified patients affected with deficiencies in factor VII, V and combined FV+FVIII. On moving to University College London, under the supervision of David Perry, we undertook genotype-phenotype characterisation initially of Iranian families with factor VII deficiency, before extending this to the rest of the world. At that time, Ted Tuddenham at the MRC and David Ginsburg at the University of Michigan, Ann Arbor, were cloning the genes that, when mutated, cause FV+FVIII deficiency. I then moved to Ken Bowers' lab in Boston to undertake in-vitro expression studies in patients with FVII deficiency.

In parallel, I was also writing my PhD, working with a large number of patients and researchers around the world to identify the clinical manifestation of each single deficiency and also to undertake molecular studies on them. Given that these are rare disorders, with a small number of patients in many different countries, this has resulted in worldwide collaborations in order to develop a strong evidence base in the form of an international database. The Rare Bleeding Disorder Database (RBDD) now includes more than 500 patients around the world.

However, at least half of the data available on rare bleeding disorders was obtained from European countries. Therefore, we decided to develop a database within Europe using a standardised methodology. The European Commission agreed to provide funding support for the three years between 2007 and 2010 to develop a network of European centres and to harmonise the data available on these disorders allowing us to write guidelines for the treatment and diagnosis of the patients. The European Network of Rare Bleeding Disorders (EN-RBD), coordinated by the University of Milan, has also been important in encouraging the pharmaceutical companies to develop products for these patients.

In all, 13 European centres from 11 countries collaborated for three years, and 489 patients have 


\section{Flora Peyvandi}

Flora Peyvandi has dedicated her entire academic and clinical career to the knowledge of the underlying causes of coagulation diseases for the purpose of determining the best medical approach to treatment for patients. She is Chair of the VWD and Rare Bleeding Disorders committee of the World Federation of Hemophilia, and member of the Executive Committee of the WFH and of the Medical Advisory Group of European Hemophilia Consortium (EHC). She is also an active member of the International Society for Thrombosis and Haemostasis. Since 1998, she has been the recipient of more than 40 project grants funded by Italian and International organisations and is leader of the prospective study of rare bleeding disorders within the European Haemophilia Network (EUHANET)

been included in this European registry. We were able, for the first time, to develop a simple and standardised classification based on four levels of bleeding severity (Table 1) and to show how the distribution of clinical bleeding severity categories differs within the different rare bleeding disorders [4]. Thus, we now know that the most severe type of rare bleeding disorders are factor XIII and fibrinogen deficiency, followed by factor X deficiency, factor VII deficiency, combined factor $V$ and factor VIII deficiency and finally factor XI deficiency. We also undertook linear regression analysis, which allowed us to identify those factor deficiencies where there is an excellent correlation between the circulating factor level and the severity of the patient (such as fibrinogen deficiency, factor X deficiency, factor XIII deficiency and combined factor $V$ and VIII deficiency), those with a good correlation (such as factor $V$ deficiency and factor VII deficiency) and those such as factor XI deficiency where there is no correlation at all.

The result of all this work has been some 70-80 publications that have shown that:

- Rare bleeding disorders are heterogeneous and need to be studied singularly

- Heterozygous patients may bleed

- There is a need to understand the minimum plasma coagulant activity level to prevent spontaneous bleeding

- Treatment of some rare bleeding disorders may be managed by using new global assays (although standardisation is required)

- Data collection on clinical history, efficacy and side effects of treatment needs to be harmonised.

Despite this, there is a lack of detailed data on the diagnosis and the chronology between diagnosis and bleeding episodes. Therefore, we are now establishing a prospective cohort study of at least three to five years that will allow us to associate "dynamic" risk factors with the outcome of bleeding and to study the efficacy of prophylaxis. The PRO-RBDD project (www.eu.rbdd.org) is a prospective cohort study on patients with coagulation, fibrinogen and factor XIII deficiencies, and the same model will be outlined in the future for the other types of disorders. It is being undertaken as part of the EUHANET project, supported by the European Commission and aimed at establishing a network of haemophilia centres to improve the care of European citizens with inherited bleeding disorders.

We have also initiated work to clarify the treatment of rare bleeding disorders by establishing a registry of products for the different factor deficiencies, whether currently available or coming soon to the market. At present, while there are plasma-derived products available for many of the rare bleeding disorders, there are only two recombinant products, which are for factor VII deficiency and factor XIII deficiency. It is hoped that in the future we will see the first factor $V$ plasma-derived product. Studies on gene therapy in haemophilia started in the late 1990s and this approach is now showing success [5-7]. A European grant application for gene therapy in severe FVII deficiency is ongoing.

\section{What's next?}

It is well known that haemophilia treatment is not equitably available worldwide. For $80 \%$ of the population there is no clinical or social network, no adequate laboratory or clinic facilities and low or absent education and training for key professionals such as haematologists, haemophilia nurses and laboratory technicians. For this reason, we have been working with centres in Afghanistan since 2011 so that clinicians can provide a minimum level of haemophilia care as set out by the World Federation of Hemophilia, by:

- Training of a haematologist and a laboratory technician at the haemophilia centre in Milan

- Opening and equipping a centre for the diagnosis and care of patients in Kabul

- Creation of a patients' association.

Since starting this work in Afghanistan in 2011, the number of diagnosed patients has risen from two to 120. A similar strategy is now being employed in Zambia. We hope that gene therapy may go some way towards solving the problems in these parts of the world.

\section{Disclosures}

The scientific meeting on which this issue of The Journal of Haemophilia Practice is based was sponsored by Baxter, Bayer, CSL Behring, Grifols, Novo Nordisk, Sobi, Pfizer, BPL and Werfen. Editorial support for the article was provided by the publisher.

\section{References}

1. European Commission. Useful information on rare diseases from an EU perspective. Available at http://ec.europa.eu/health/ph_information/documents/ev20040705_ rd05_en.pdf

2. World Federation of Hemophilia. Annual Global Survey, 2013. Available at http:// www1.wfh.org/publications/files/pdf-1591.pdf

3. Peyvandi F, Palla R, Menegatti M, Mannucci PM. Introduction. Rare bleeding disorders: general aspects of clinical features, diagnosis, and management. Semin Thromb Hemost 2009; 35: 349-55.

4. Peyvandi F, Palla R, Menegatti M, et al. Coagulation factor activity and clinical bleeding severity in rare bleeding disorders: results from the European Network of Rare Bleeding Disorders. J Thromb Haemost 2012; 10(4): 615-21. doi: 10.1111/j.15387836.2012.04653.x.

5. Nathwani AC, Tuddenham EG, Rangarajan S, et al. Adenovirus-associated virus vector-mediated gene transfer in hemophilia B. N Engl J Med 2011;365(25): 2357-65 doi: 10.1056/NEJMoa1108046.

6. Ward NJ, Buckley SM, Waddington SN, et al. Codon optimization of human factor VIII cDNAs leads to high-level expression. Blood 2011;117(3):798-807. doi: 10.1182/ blood-2010-05-282707.

7. Mclntosh J, Lenting PJ, Rosales C, et al. Therapeutic levels of FVIII following a single peripheral vein administration of rAAV vector encoding a novel human factor VIII variant. Blood 2013; 121(17): 3335-44. doi: 10.1182/blood-2012-10-462200. 